\section{Immunohistochemical/ histochemical double staining method in the study of the columnar metaplasia of the oesophagus}

\author{
D. Cabibi, ${ }^{1}$ A.G. Giannone, ${ }^{1}$ C. Mascarella, \\ C. Guarnotta, ${ }^{1}$ M. Castiglia, ${ }^{2}$ \\ G. Pantuso, ${ }^{2}$ E. Fiorentino ${ }^{2}$
}

'Department of Science for Promotion of Health and Mother and Child Care

“G. D'Alessandro", University of Palermo

2Department of Surgical, Oncological

and Stomatological Disciplines,

University of Palermo, Italy

\begin{abstract}
Intestinal metaplasia in Barrett's oesophagus (B0) represents an important risk factor for oesophageal adenocarcinoma. Instead, few and controversial data are reported about the progression risk of columnar-lined oesophagus without intestinal metaplasia (CLO), posing an issue about its clinical management. The aim was to evaluate if some immunophenotypic changes were present in CLO independently of the presence of the goblet cells. We studied a series of oesophageal biopsies from patients with endoscopic finding of columnar metaplasia, by performing some immunohistochemical stainings (CK7, p53, AuroraA) combined with histochemistry (Alcian-blue and Alcian/PAS), with the aim of simultaneously assess the histochemical features in cells that shows an aberrant expression of such antigens. We evidenced a cytoplasmic expression of CK7 and a nuclear expression of Aurora A and p53, both in goblet cells of $\mathrm{BO}$ and in non-goblet cells of CLO, some of which showing mild dysplasia. These findings suggest that some immunophenotypic changes are present in CLO and they can precede the appearance of the goblet cells or can be present independently of them, confirming the conception of BO as the condition characterized by any extention of columnar epithelium. This is the first study in which a combined immunohistochemical/histochemical method has been applied to Barrett pathology.
\end{abstract}

\section{Introduction}

Barrett's oesophagus (B0) was endoscopically defined by the presence of at least 2 to 3 $\mathrm{cm}$ of columnar epithelium in the lower oesophagus. Recently, the Global EvidenceBased Consensus of Montreal defined Barrett oesophagus as columnar metaplasia lining the distal oesophagus, with specialized intestinal metaplasia (IM) with goblet cells, or gastric metaplasia with cardial type or fundic-oxyntic type mucosa. ${ }^{1}$ The main feature of intestinal metaplasia is the presence of Alcian blue-positive mucin-producing goblet cells. Moreover, in clinical practice, the finding of cardial and/or fundic mucosa without intestinal metaplasia ${ }^{2-4}$ in the distal oesophagus is frequent and it has been reported as columnar-lined oesophagus (CLO). However, it probably represents an early reflux-lesion preceding an evident Barrett phenotype with goblet cells, in keeping with previous studies showing that CK7 is a marker related to early phase of gastroesophageal reflux, and it is present also in CLO biopsies lacking of IM. ${ }^{5} \mathrm{BO}$ with IM represents an important risk factor for oesophageal adenocarcinoma, in keeping with the progression sequence metaplasia-dysplasia-adenocarcinoma. Chandrasoma et al. hypothesized that patients with cardial metaplasia could potentially progress towards dysplasia and adenocarcinoma through IM.-7 Instead, few and controversial data are reported in the literature about the progression risk of CLO lacking of IM, giving rise to a question about the clinical management of this lesion. On this basis, we studied a series of oesophageal biopsies from patients with endoscopic abnormalities related to columnar metaplasia, by performing some double and triple immunohistochemical/histochemical stains (CK7/Alcian/PAS, p53/Alcian/ PAS, Aurora A/Alcian/PAS) in order to simultaneously assess the mucinous histochemical features of the cells with aberrant expression of such antigens. The aim of the study was to evaluate if some immunophenotypic changes were present in CLO cases without IM and if these changes could precede the appearance of the goblet cells or may be present independently of them. Aurora A maps in 20q13.2, a chromosomal region frequently amplified and overexpressed in several human malignancies (breast, bladder, prostate, ovary, colon and pancreas). ${ }^{8}$ Recently its expression was evidenced in Barrett adenocarcinoma and in CLO.,10 In experimental studies, the over-expression of Aurora A was oncogenic and constantly associated with mutation of the gene $T p 53^{11,12}$ that, becoming unable to block the kinase activity of the Aurora A, leads to a strong accumulation of extra-copies of centrosomes. ${ }^{13}$ Cells without a functional p53 and with high levels of Aurora A are characterized by repeated cell cycles, until they become aneuploid. These observations identify the protein Aurora A as a prognostic marker, being itself an accomplice of carcinogenesis. To our knowledge, this is the first study in which a combined immunohistochemical/ histochemical method has been applied in the study of Barrett pathology.
Correspondence: Dr. Antonino Giulio Giannone Dipartimento di Scienze per la Promozione della Salute e Materno-Infantile "G. D’Alessandro", Anatomia Patologica, A.0.U. Policlinico "P. Giaccone”, via del Vespro 129, 90127 Palermo, Italy.

Tel. +39.91.6553529 - Fax: +39.91.6553549.

E-mail: aggianno@alice.it

Keywords: Barrett's oesophagus, columnar-lined oesophagus, p53, Aurora A kinase.

Contributions: DC, study conception and design, data interpretation, manuscript drafting; AGG, data analysis, manuscript drafting; CM, CG, data aquisition, manuscript drafting; GP, EF, study design contribution and critical revision for important intellectual content.

All the authors approved the version to be published.

Conflict of interests: the authors have no financial relationships nor conflict of interests to disclose.

Received for publication: 3 July 2013.

Accepted for publication: 23 January 2014.

This work is licensed under a Creative Commons Attribution NonCommercial 3.0 License (CC BYNC 3.0).

CCopyright D. Cabibi, et al., 2014

Licensee PAGEPress, Italy

European Journal of Histochemistry 2014; 58:2326 doi:10.4081/ejh.2014.2326

\section{Materials and Methods}

This is a retrospective study performed on 57 biopsies obtained from $H$. pylori-free patients showing columnar metaplasia of the lower oesophagus at the endoscopy. The consent for study participation was obtained from all patients. The study was approved by the Institutional Ethic Committee. The cases were retrieved from broader clinical records, consisting of 819 oesophageal biopsies collected between June 2006 and June 2012 and comprising 228 cases (27.8\%) of columnar metaplasia (135 with BO and 93 with CLO) and 26 cases of adenocarcinoma (3.1\%).

Formalin-fixed, paraffin-embedded specimens were retrieved from the Archives of the Department of Pathology, University of Palermo (Italy). For each patient, 4-quadrant biopsy specimens have been taken every $2 \mathrm{~cm}$ and stained with Hematoxylin-Eosin and Alcian-PAS. The patients were divided into three groups: i) Group A, 20 BO cases consisting of IM, with Alcian blue-positive goblet cells; ii) Group B, 17 cases of CLO consisting of PAS+ columnar metaplasia without Alcian blue positive goblet cells; iii) Group C, 20 cases 
with intermediate features of transitional metaplasia (TM), consisting of PAS + columnar mucosa with only few cells showing initial production of acid-Alcian blue positive-mucins, but devoid of goblet cell morphology. All the cases were stained with combined double/triple immunohistochemical/histochemical methods. The combined staining method consists of two steps: in the first step an immunohistochemical staining was performed, according to the manufacturer's instructions (Universal LSAAB kit, Dako). Primary antibodies used in this study were: CK7 (Clone SP52; 1:100; Ventana), p53 (Clone D07; 1:200; Ventana) and Aurora A (Clone EP1008Y; 1:100; Epitomics). Immunohisto chemical positivity was considered significant when it was cytoplasmic for CK7, nuclear for p53 and nuclear or nuclear/cytoplasmic for Aurora A. As positive control for Aurora A and p53, we referred to a case of adenocarcinoma arising in the lower oesophagus (Barrett adenocarcinoma). For Aurora A and p53, the positivity in at least $3 \%$ of the nuclei was considered significant. For CK7, cytoplasmic staining in at least one gland was considered significant. Negative controls, lacking primary antibody incubations, were included for each immunohistochemical run.

In the second step, a histochemical staining was performed on the same sections previously subjected to the immunohistochemical assay. At first, Alcian blue staining was performed and the results of this double immunohistochemical/histochemical staining were observed. Subsequently, PAS staining was added (triple staining) and the results were observed again. The histochemical stainings were performed with only minor modifications according to Poletti et al. ${ }^{14}$ For Alcian blue staining, the sections were stained with Alcian blue solution ( $\mathrm{pH} \mathrm{2.5)}$ for $30 \mathrm{~min}$ at room temperature, washed in running tap water for 10 min, rinsed in DI water, counterstained in Mayer's hematoxylin for $5 \mathrm{~min}$ and washed in DI water. The acetic acid as mordant was not used because it has no effect on the final results. For PAS staining, sections were oxidized in $0,5 \%$ periodic acid solution for $5 \mathrm{~min}$, rinced in DI water, placed in Schiff reagent for $15 \mathrm{~min}$, washed in tap water for $5 \mathrm{~min}$, counterstained in Mayer's hematoxylin for $1 \mathrm{~min}$ and washed in DI water. Finally, the slides were dehydrated, cleared and mounted. Differentiation with hydrochloric acid was omitted because it markedly reduces tissue antigenicity and causes fading of the chromogenic reaction when the immunohistochemical staining is performed before PAS staining.

\section{Results}

A case of adenocarcinoma of the lower oesophagus (Barrett adenocarcinoma) is showed in Figure 1a. The tumor exhibited strong nuclear positivity for p53 and Aurora A (Figure 1b) and it was used as positive control for the immunohistochemical stains with these antibodies. CK7 was positive in all the three groups; it was more diffusely and strongly expressed in group $\mathrm{A}$, while focally and more weakly in groups $\mathrm{B}$ and $\mathrm{C}$.

Group A. Both Aurora A and p53 immunostainings were positive in the nuclei of Alcian blue+ goblet cells in 6 of 20 cases (Figure $2 a$ ). Noteworthy, they were positive also in nuclei of PAS+/Alcian blue negative columnar cells, whereas adjacent Alcian blue+ goblet cells were negative [Figure 2b].

Group B. Eight of 17 cases showed focal nuclear positivity for Aurora A and p53 in PAS+/Alcian blue negative columnar cells (Figure 3 b,c). Noteworthy, CK7 was expressed just in the same Aurora A/p53 positive areas (Figure 3d).
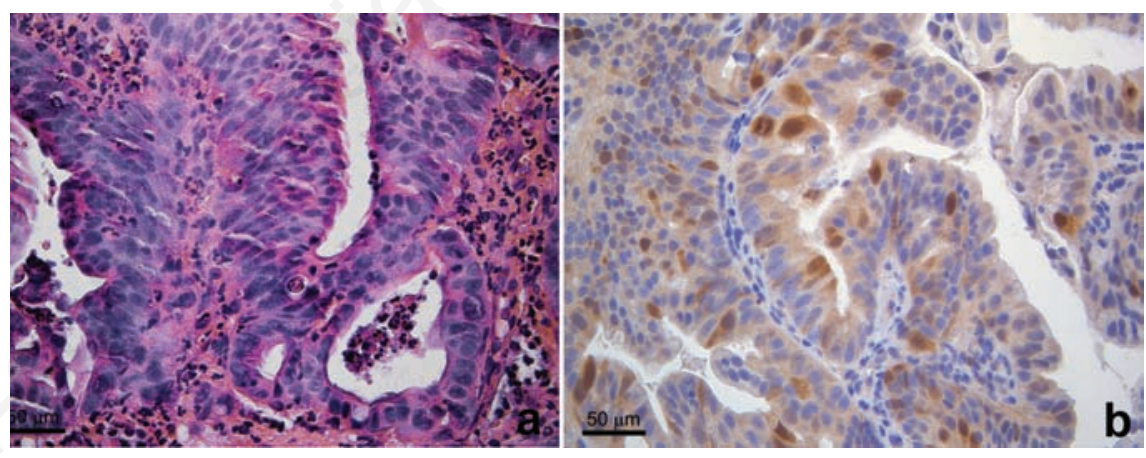

Figure 1. Adenocarcinoma of the oesophagus. a) Hematoxylin and eosin stain. b) Aurora A immunohistochemical stain. Strong nuclear positivity in neoplastic cells.

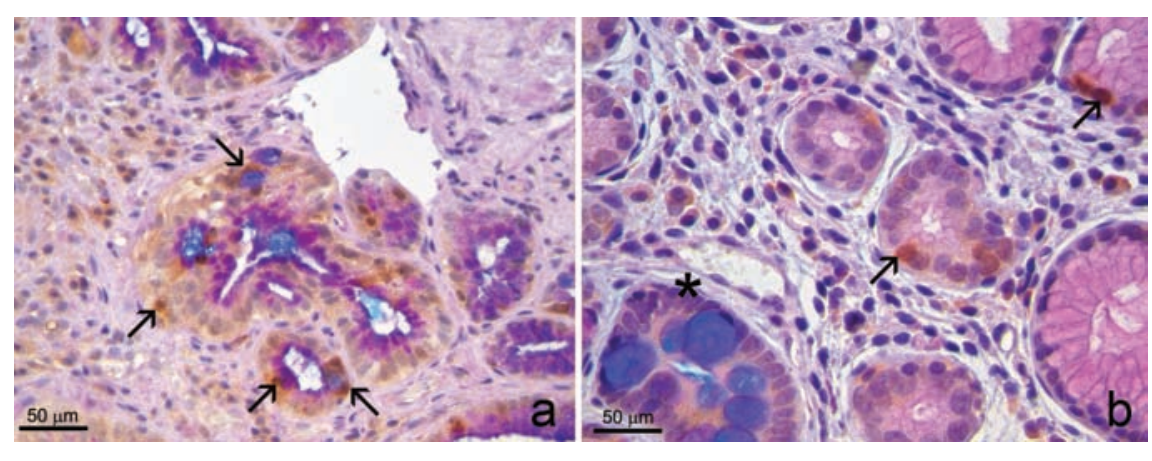

Figure 2. Group A. Intestinal metaplasia with goblet cells. a) Positivity for Aurora A in nuclei of glands comprising Alcian blue+ goblet cells (arrows). b) In another field of the same case, Aurora A was positive only in nuclei of Alcian blue-/PAS + columnar cells (arrows), whereas the gland with Alcian blue+ goblet cells was negative (asterisk). a,b) Triple Aurora A/Alcian blue/PAS stain. 


\section{Discussion}

The combined use of immunohistochemical/histochemical staining methods has rarely been reported in the literature. Wong et al. used the double Alcian blue/Chromogranin A and Alcian-blue/Mib-1 staining in specimen of gastric mucosa with intestinal metaplasia and neuroendocrine hyperplasia, to distinguish the proliferating cellular component. ${ }^{15}$ To our knowledge, a double histochemical/immunohistochemical method has not been applied to the study of oesophageal pathology.

In our study, all the cases stained positively, at least focally, for CK7, considered an early marker of gastroesophageal reflux because it is usually absent in normal fundic and cardial mucosa, but present in columnar metaplastic mucosa of the oesophagus, including cases lacking of IM. ${ }^{5}$ Furthermore, our study showed nuclear expression of Aurora A kinase in columnar metaplasia lacking of goblet cells (group B), often correlating with p53 nuclear expression. The Aurora A kinase belongs to serine/threonine group of kinases that operate as key regulators of mitosis and it has a role in the cell cycle progression. Several studies showed that a deregulation in the expression and activity of this protein leads to defects in the assembly of the centrosome and of the mitotic spindle, aneuploidy, genetic instability

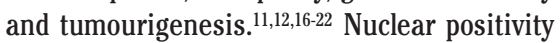
has been reported in hormone-related (breast, ovary and prostate) human cancers associated with a poor outcome, ${ }^{23,24}$ but not in non-hormone responsive conditions, such as in the oesophageal columnar metaplasia and in the Barrett adenocarcinoma. Previously, we evidenced the overexpression of Aurora A with RT-PCR in cases of columnar mucosa with and without IM. ${ }^{10}$

In the present study, we evidenced Aurora A immunohistochemical overexpression in the nuclei of PAS+ cells. In the group A we found nuclear Aurora A-positive staining in some Alcian blue+ goblet cells of Barrett mucosa, but, noteworthy, other goblet cells did not stain for Aurora A, whereas the adjacent PAS+ columnar cells stained positively. This is in keeping with the findings of Group B cases, lacking of IM, in which Aurora A was present in the nuclei of PAS+ columnar cells.

The over-expression of Aurora A in non-goblet cells may support the recently introduced conception of BO without intestinal metapla$s i a,{ }^{1}$ in contrast with the definition in which the goblet cells represented an essential prerequisite for the diagnosis of $\mathrm{BO}$ and an obliged step in the cancer progression. In fact, the definition of BO is still confusing and ambiguous. According to the American College of Gastroenterology $y^{25}$ and to the American
Gastroeneterological Association, ${ }^{26}$ intestinal metaplasia is the only type of oesophageal columnar epithelium that clearly predisposes to malignancy and it is still required for the diagnosis of B0. Furthermore, our findings are in keeping with Kelty et al., who observed that patients with CLO lacking of IM on biopsy have a similar cancer risk to those with specialized IM, so they conclude that IM is not essential for cancer risk. ${ }^{27}$ Of course, since the carcinogen-

esis is a multistep process consisting of many overlapping mutations, the over-expression of Aurora A in the columnar mucosa, with or without IM, should not be considered a condition necessarily leading to the tumour, but a potentially reversible condition after stopping or decreasing of the stimulus. The appearance of Aurora A and p53 nuclear positivity, together with the reflux-related CK7 cytoplasmic positivity, in areas lacking of IM, could suggest
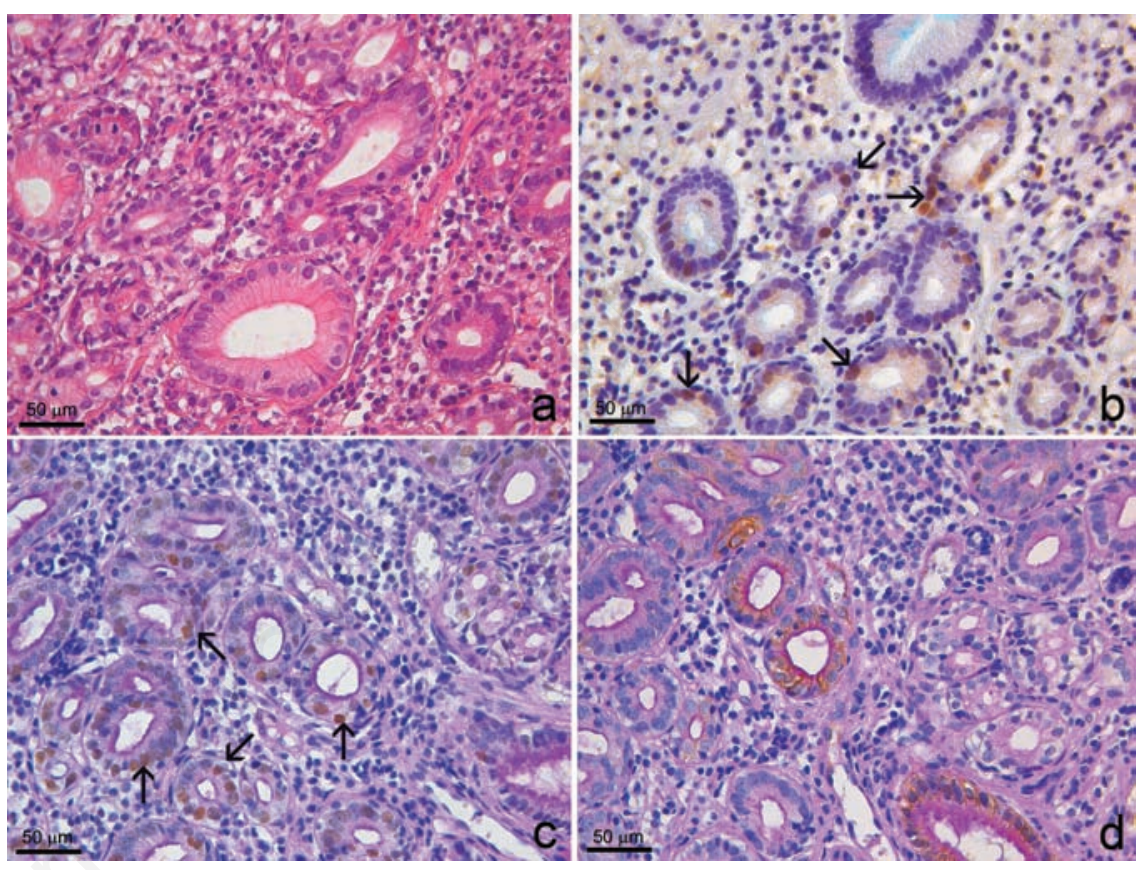

Figure 3. Group B. Columnar lined oesophagus. a) Hematoxylin and eosin stain. b) Double Aurora A/Alcian blue stain showed Alcian blue negativity and Aurora A nuclear positivity (arrows). c) Triple p53/Alcian blue/PAS stain showed cytoplasmic PAS positivity and 53 nuclear positivity (arrows). d) Triple Ck7/Alcian blue/PAS stain shows Ck7 positivity in Alcian blue-/PAS+ cells.
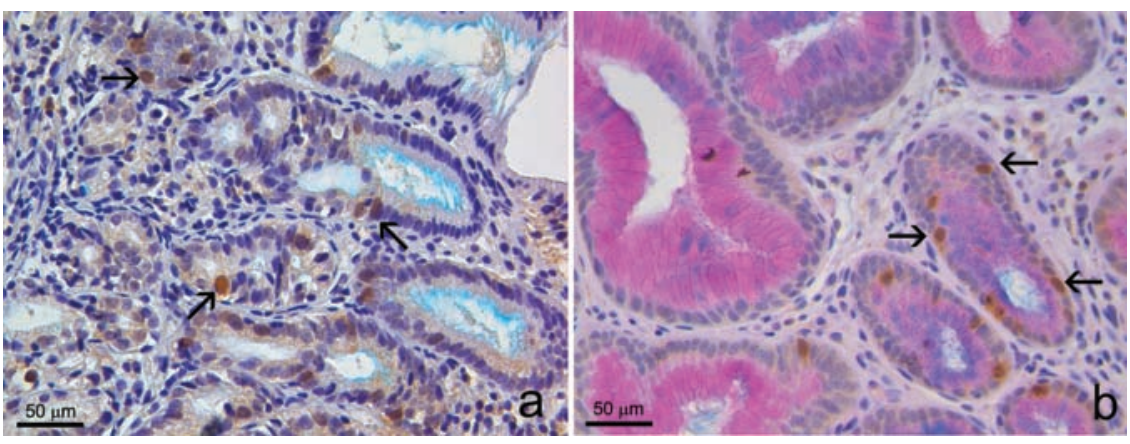

Figure 4. Group C. Mucosa with a few cells with intermediate features between intestinal metaplasia and columnar-lined oesophagus, showing initial production of Alcian blue positive mucins, but lacking of goblet cell morphology. a) Aurora A positivity both in Alcian blue negative cells (arrows); double Aurora A/Alcian blue stain. b) Aurora A positivity in cells with initial production of acid mucins (arrows); triple Aurora A/Alcian blue/PAS stain). 


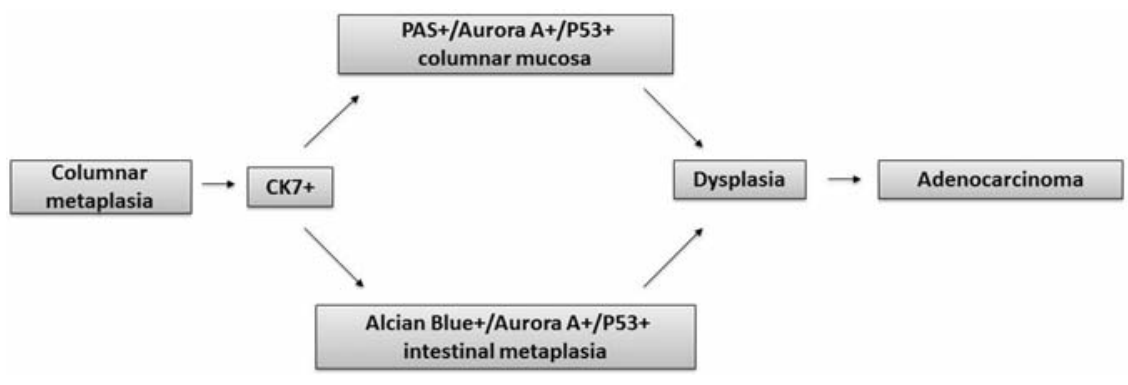

Figure 5. Cancer sequence hypothesis, in which $\mathrm{Ck} 7 /$ AuroraA/p53/PAS+ columnar mucosa could represent an intermediate step of the carcinogenesis, independently from the presence of intestinal Alcian blue+ goblet cells.
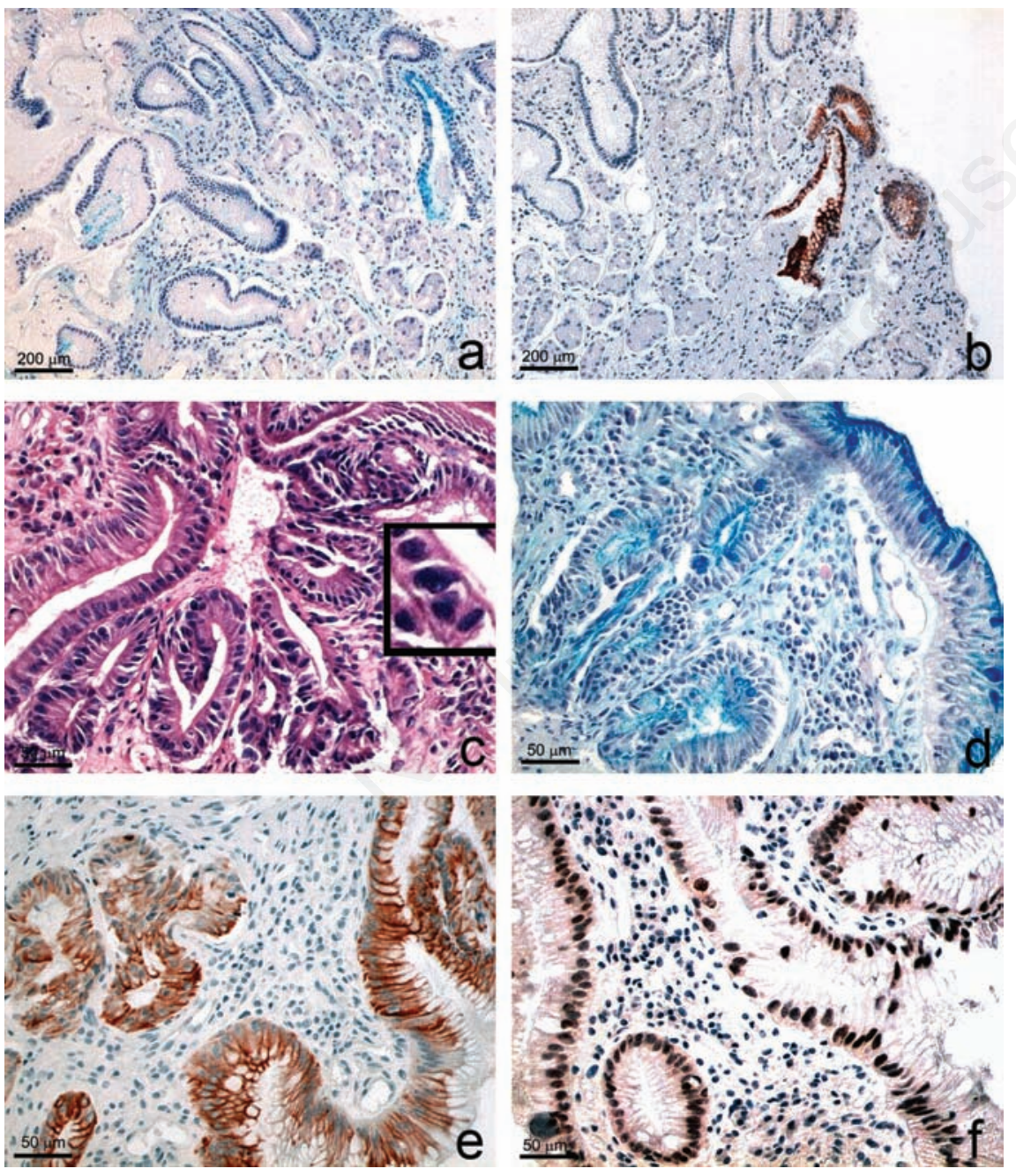

Figure 6. Progression from columnar-lined oesophagus to Barrett's oesophagus with dysplasia in a patient never treated with PPI. a-b) Baseline observation: weak Alcian positivity (a) and very focal $\mathrm{Ck} 7$ positivity (b), in a few columnar cells of cystically dilated glands (a, Alcian PAS; b, Ck7). c-f) Two years follow-up observation of the same case, showing dysplasia (c) intestinal metaplasia (d), diffuse cytoplasmic CK7 (e) and nuclear p53 immunostaining (f). The insert in c) shows nuclear changes and mitoses. c) H-E; d) Alcian PAS; e) CK7; f) p53. another way besides the traditional IM-dysplasia-adenocarcinoma sequence. In keeping with our immunophenotypical findings, we hypothesize a cancer sequence, in which CK7/ AuroraA/p53/PAS+ columnar mucosa could represent an intermediate step of the carcinogenesis, independently from the presence of intestinal Alcian blue+ goblet cells (Figure 5). Recently, in fact, adenocarcinoma of gastroesophageal junction arising from cardial type metaplasia have been described, ${ }^{28}$ suggesting again the hypothesis of a double pathway previously reported by us. ${ }^{5}$

Noteworthy, in our study, consisting of 819 oesophageal biopsies collected between June 2006 and June 2012, we found 26 cases of adenocarcinoma (3.1\%), 25 of which aroused in patients without a known clinical history of B0, lacking previous endoscopy and never treated with GERD therapy. On the contrary, none of 228 patients with endoscopic and histologic columnar-lined oesophagus (135 with B0 and 93 with CLO), treated with constant therapy for GERD symptoms, developed a high-grade dysplasia/adenocarcinoma, during a mean followup of 5 years (range 1-12) with bi-annual endoscopy and biopsy. Only one case of CLO, untreated, progressed to $\mathrm{BO}$ with dysplasia (Figure 6). Given our results, we think that the immunophenotypical alterations (such as Aurora A and p53 expression) in some cells of CLO cases could be by themselves responsible of a risk progression, despite the absence of IM. Therefore, in our view, also CLO patients require an appropriate management, because the outcome is not $a b$ initio predictable. Of course, in consideration of the low risk of cancer progression, both in CLO and BO patients treated with PPI therapy, and of the necessity to refine the surveillance program to obtain a good cost/benefit ratio, we think that a more longterm interval of endoscopies (e.g., a 3-year interval) could be enough if $\mathrm{BO}$ and $\mathrm{CLO}$ cases are properly treated. PPI therapy, in fact, could be useful not only for the GERD symptoms but also to reduce the risk of cancer progression.

In conclusion, the combined staining method allowed us to simultaneously evidence the type of mucins produced by the columnar cells and the immunophenotypical expression of Aurora A, p53 and CK7 that may be indicative of an early phase of the carcinogenetic pathway independently from the IM step.

\section{References}

1. Vakil N, van Zanten SV, Kahrilas P, Dent J, Jones R, et al. The Montreal definition and classification of gastroesophageal reflux disease: global evidence-based consensus. Am J Gastroenterol 2006;101:1900-20. 
2. Haggitt RC. Barrett's esophagus, dysplasia, and adenocarcinoma. Hum Pathol 1994;25:982-93.

3. Blot WJ. Esophageal cancer trends and risk factors. Semin Oncol 1994;21:403-10.

4. Jankowski JA, Wright NA, Meltzer SJ, Triadafilopoulos G, Geboes K, Casson AG, et al. Molecular evolution of the metaplasia-dysplasia-adenocarcinoma sequence in the esophagus. Am J Pathol 1999;154: 965-73.

5. Cabibi D, Fiorentino E, Pantuso G, Mastrosimone A, Callari C, Cacciatore M, et al. Keratin 7 expression as an early marker of reflux-related columnar mucosa without intestinal metaplasia in the esophagus. Med Sci Monit 2009;15:CR203-10.

6. Chandrasoma PT, Der R, Dalton P, Kobayashi G, Ma Y, Peters J, DeMeester T. Distribution and significance of epithelial types in columnar-lined esophagus. Am J Surg Pathol 2001;25:1188-93.

7. Chandrasoma P. Pathophysiology of Barrett's esophagus. Semin Thorac Cardiovasc Surg 1997;9:270

8. Karthigeyan D, Prasad SB, Shandilya J, Agrawal S, Kundu TK. Biology of Aurora A kinase: Implications in cancer manifestation and therapy. Med Res $\operatorname{Rev} 2010 ; 31$ : 757-93.

9. Rugge M, Fassan M, Zaninotto G, Pizzi M, Giacomelli L, Battaglia G, et al. Aurora kinase A in Barrett's carcinogenesis. Hum Pathol 2010;41:1380-6.

10. Agnese V, Cabibi D, Calcara D, Terrasi M, Pantuso G, Fiorentino E, et al. Aurora-A overexpression as an early marker of reflux-related columnar mucosa and Barrett's esophagus. Ann Oncol 2007;18:vil10-5.

11. Fu J, Bian M, Jiang Q, Zhang C. Roles of
Aurora kinases in mitosis and tumorigenesis. Mol Cancer Res 2007;5:1-10.

12. Marumoto T, Zhang D, Saya H. Aurora A-a guardian of poles. Nat Rev Cancer 2005;5:42-50.

13. Meraldi P, Honda R, Nigg EA. Aurora-A overexpression reveals tetraploidization as a major route to centrosome amplification in p53-/- cells. EMBO J 21:483-92.

14. Poletti A, Giacon, C Pennelli N Simultaneous visualization of immunodetected antigens and tissue components revealed by nonenzymatic histochemical stains. J Histochem Cytochem 1992;40: 1965-70.

15. Wong WM, Stamp GWH, Elia G, Poulsom R, Wright NA. Proliferative populations in intestinal metaplasia: evidence of deregulation in Paneth and goblet cells, but not endocrine cells. J Pathol 2000;190:107-13.

16. Carmena M, Earnshaw WC. The cellular geography of aurora kinases. Nat Rev Mol Cell Biol 2003;4:842-54.

17. Descamps S, Prigent C. Two mammalian mitotic aurora kinases: who's who? Sci STKE 2001;73:pe1.

18. Mountzios G, Terpos E, Dimopoulos MA. Aurora kinases as targets for cancer therapy. Cancer Treat Rev 2008;34:175-82.

19. Carvajal RD, Tse A, Schwartz GK. Aurora kinases: new targets for cancer therapy. Clin Cancer Res 2006;12:6869-75.

20. Giet R, Petretti C, Prigent C. Aurora kinases, aneuploidy and cancer, a coincidence or a real link? Trends Cell Biol 2005;15: 241-250.

21. Burum-Auensen E, De Angelis PM, Schjolberg AR, Kravik KL, Aure M, Clausen OP. Subcellular localization of the spindle proteins Aurora A, Mad2, and BUBR1 assessed by immunohistochemistry. J
Histochem Cytochem 2007;55:477-86.

22. Chng WJ, Braggio E, Mulligan G, Bryant B, Remstein E, Valdez R et al. The centrosome index is a powerful prognostic marker in myeloma and identifies a cohort of patients that might benefit from aurora kinase inhibition. Blood 2008 1;111:1603-9.

23. Das K, Lorena PD, Ng LK, Shen L, Lim D, Siow WY, et al. Aurora-A expression, hormone receptor status and clinical outcome in hormone related cancers. Pathology 2010;42:540-6.

24. Yang F, Guo X, Yang G, Rosen DG, Liu J. AURKA and BRCA2 expression highly correlate with prognosis of endometrioid ovarian carcinoma. Mod Pathol 2011;24: 836-45.

25. Wang KK, Sampliner RE. Updated guidelines 2008 for the diagnosis, surveillance and therapy of Barrett's esophagus. Am J Gastroenterol 2008;103:788-971.bis

26. American Gastroenterological Association, Spechler SJ, Sharma P, Souza RF, Inadomi JM, Shaheen NJ. American Gastroenterological Association medical position statement on the management of Barrett's esophagus. Gastroenterology 2011;140: 1084-91.

27. Kelty CJ, Gough MD, Van Wyk Q, Stephenson TJ, Ackroyd R. Barrett's oesophagus: intestinal metaplasia is not essential for cancer risk. Scand J Gastroenterol 2007;42:1271-4.

28. Demicco EG, Farris AB 3rd, Baba Y, AgborEtang B, Bergethon K, Mandal R, et al. The dichotomy in carcinogenesis of the distal esophagus and esophagogastric junction: intestinal-type vs cardiac-type mucosaassociated adenocarcinoma. Mod Pathol 2011;24:1177-90. 